\title{
Immunosuppression and Cancer
}

\author{
By Israel Penn and Thomas E. Starzl
}

B ESIDES their beneficial effect in retarding or arresting the growth of certain types of neoplasms, a number of anticancer drugs may cause impairment of antibody synthesis and cell-mediated immunity. These immunosuppressive effects have been used therapeutically to prevent and control rejection of organ homografts and also to treat a variety of clinical states. However, the anticancer agents have mutagenic, teratogenic, and oncogenic properties in experimental animals and presumably also in humans. The present report is concerned with their potential oncogenic effects in man.

Evidence has been accumulated from three groups of patients about which full details are being published in Cancer. ${ }^{1}$ It should be emphasized that the vast majority of the cases have been contributed to us or have appeared in the literature and did not come from our own center. The groups include (1) immunosuppressed organ transplant recipients; (2) patients with a variety of noncancerous diseases treated with immunosuppressive agents; (3) Patients with malignant tumors who received cancer chemotherapy.

\section{ORGAN TRANSPLANT RECIPIENTS}

Since 1968 we have maintained an informal Tumor Registry in Denver to record

From the Department of Surgery, University of Colorado School of Medicine and the Veterans Administration Hospital, Denver, Colorado.

Supported by research grants from the Veterans Administration, by grants RR-0005I and RR-00069 from the General Clinical Research Centers program of the Division of Research Resources, National Institutes of Health, and by grants AI-10176-01, AI-AM-08898, and AM-07772 of the United States Public Health Service.

(C) 1973 by Grune \& Stratton, Inc. cases of cancer in organ homograft recipients. $^{1-4}$ Tumors were encountered in three groups of transplant patients. (1) those which arose de novo after transplantation; (2) those inadvertently transmitted with the homograft; and (3) those which were present before transplantation.

Cancers Which Appeared After Transplantation: An organ transplant recipient maintained on chronic immunosuppressive therapy has a $5-6 \%$ chance of developing a de novo cancer within the first few years after transplantation. ${ }^{1-4}$ We have collected details of 122 such cases from transplant centers throughout the world. The patients had 125 tumors of which 76 were of epithelial origin $(61 \%)$ and $49(39 \%)$ were mesenchymal.

The most common epithelial lesions were various skin cancers (27 cases, 36\%), carcinomas of the cervix (11 cases, $14 \%$ ) and carcinomas of the lip (11 cases, 14\%). The remainder consisted of a wide variety of visceral carcinomas, many of high-grade malignancy.

Forty-two ( $86 \%$ ) of the 49 mesenchymal tumors were solid lymphomas, of which the most prominent subgroup was reticulum cell sarcoma (30 cases, $61 \%$ ). A most unusual feature of the lymphomas was their predeliction for the central nervous system which occurred in 20 of 41 cases $(49 \%)$.

The cancers occurred at an average age of $36 \mathrm{yr}$. The mean time of appearance of the tumors after transplantation was 28 mo (range 1-92 mo). The possibility of transplantation of cancer from the donors was very small as only three of the 137 donors had tumors. Two of the three were cadaver donors who had medulloblastomas. The third donor, also a cadaver, had had a 
carcinoma of the colon resected $5 \mathrm{yr}$ previously and was apparently free of cancer at the time of donation. The recipients subsequently developed apparently unrelated cancers-reticulum cell sarcoma in two instances and a leiomyosarcoma in one.

Almost all of the patients received immuno suppression with Azathioprine and prednisone. Other immunosuppressive measures used were ALG (38), actinomycin (39), roentgen therapy to the homograft (45), splenectomy (41), thymectomy (7), thymic irradiation (2), thoracic duct lymph drainage (7), cyclophosphamide (3), endolymphatic radiation (1), total body irradiation (1), methotrexate (1), 6-mercaptopurine (1), and azaserine (1).

Treatment of the epithelial lesions of skin, lip, and uterine cervix followed conventional lines and was usually successful. Other epithelial tumors had a worse prognosis and in most instances either caused or contributed to the patients' deaths. The overall survival in patients with epithelial cancers was 44 of $74(59 \%)$.

The outlook for recipients with mesenchymal neoplasms was more gloomy as only 11 of 48 patients $(23 \%)$ are still living. Experience thus far is limited but it appears that conventional cancer therapy combined with reduction or cessation of immunosuppression may permit the patient's immune system to recover and resist the neoplasm. Five of the current survivors with highly malignant tumors were treated in this way with apparent eradication of the lesion and two more patients who died of infection or homograft failure were found to be free of cancer at autopsy.

\section{Transplanted Cancers}

Thirty-three patients received kidneys removed from donors who had cancer at the time of donation or who manifested evidence of the disease some months afterward. Subsequently, 19 recipients showed no evidence of tumor either at autopsy or during follow-up from 1-32 mo. Presumably these last kidneys were either free of cancer or transplanted malignant cells failed to become established in the host. In four patients, tumor was found in homografts removed within the first 16 days after transplantation. Two more recipients developed involvement by cancer of the kidney and adjacent structures, while eight additional patients also had evidence of distant spread. Five of these last eight patients died of the transplanted cancer and in the remaining three patients immunosuppression was discontinued and the neoplasms apparently underwent rejection. One is still well 97 mo posttransplantation despite further immunosuppressive therapy given for two subsequent renal transplants each of which functioned for 12-18 mo. The other two patients died several months after cessation of immunosuppression and no tumor was present at autopsy.

\section{Cancers Which Were Present Before Transplantation}

Fifty-three organ recipients had cancers within the $5 \mathrm{yr}$ preceding transplantation. In 14 instances the tumor did not involve the organ undergoing replacement; while in 39 cases transplantation was performed for treatment of cancer of one or both kidneys (21 cases), primary or metastatic cancer of the liver (17 cases), and carcinoma of the larynx (one case). When transplantation was performed in the treatment of cancer the neoplasm appeared to be localized and resectable so that there was hope of obtaining a "cure."

Of the 53 recipients, $28(53 \%)$ had no evidence of tumor in follow-up of 2-42 mo. Twenty-two patients $(41 \%)$ developed recurrent or metastatic cancers, and three $(6 \%)$ developed de novo tumors of a type completely different from the original neoplasms. 


\section{PATIENTS WITH NONCANCEROUS DISEASES TREATED WITH IMMUNOSUPPRESSIVE AGENTS}

Thirty patients suffering from chronic cold hemagglutinin disease, rheumatoid arthritis, the nephrotic syndrome, systemic lupus erythematosus, ulcerative colitis, or psoriasis were treated with immunosuppressive agents and developed cancer. It might be argued that the immunosuppressive agents played no role in the development of the tumors in many of these disorders as they are autoimmune diseases in which an increased incidence of cancer has been reported. However, this argument does not apply to psoriasis which is not usually associated with cancer. The development of cancer in 20 psoriatic patients chronically treated with methotrexate or aminopterin must, therefore, be regarded with the gravest suspicion.

\section{PATIENTS WITH CANCERS WHO} RECEIVED CANCER CHEMOTHERAPY

Sixty-one patients received cancer chemotherapy for one type of neoplasm and subsequently developed a new cancer of a different type. Were the anticancer drugs the cause of the tumors or could these have occurred spontaneously? It is well recognized that a patient with one type of cancer is more prone to develop a second neoplasm. Furthermore, certain tumor associations are widely accepted such as the relationship between solid lymphoma and lymphocytic leukemia or the termination of chronic myelogenous leukemia in acute myeloblastic leukemia. A few of the 61 cases may have been of this type. However, certain associations are decidedly uncommon and raise the strong suspicion that the cancer chemotherapeutic agents, while controlling the original neoplasm, may have contributed to the development of the second type of tumor. A strikingly example was the development of acute leukemia in
21 patients with multiple myeloma who were chronically treated with anticancer drugs, most commonly melphalan. Another is the development of a solid lymphoma in nine cases of chronic granulocytic leukemia. There are numerous additional cases in which a second tumor appeared while the patient was receiving chemotherapy for cancer. In many of these reports the authors raised the question whether the second neoplasm was induced by the very agent which had controlled the first cancer.

\section{DISCUSSION}

In animal studies numerous experiments have elicited the paradox that agents which can destroy or arrest the growth of cancer may themselves be oncogenic. Do the anticancer and immunosuppressive agents cause cancer in man, and if so, by what mechanism? What is the effect of these agents on existing cancers?

The answer to the first question is provided mainly by experience with organ homograft recipients. We have repeatedly reported a $5-6 \%$ incidence of de novo cancers in organ homograft recipients treated with chronic immunosuppressive therapy. ${ }^{1-4}$ These findings are reinforced by experience with neoplasms inadvertently transplanted with kidneys obtained from donors with cancer. It is very rarely possible to transplant cancer cells successfully. from one healthy human to another as they are recognized as "foreign" by the host's defenses and are readily destroyed. However, if the normal defense mechanisms are impaired by chronic immunosuppression, it is possible for the transferred malignant cells to become established in the homograft, invade the surrounding tissues, and metastasize widely. If the immunosuppressive therapy is discontinued, the immune defenses may recover and reject the cancer cells. This approach was successfully used in several cases reported in this paper. 
Furthermore, it may also be applicable to the management of the more aggressive de novo tumors which arise posttransplantation and which fail to respond to conventional cancer therapy.

The concept that tumors may arise in individuals under chronic immunosuppressive therapy is further strengthened by reports concerning nontransplant patients treated with these agents. This applies particularly to sufferers from psoriasis who received chronic treatment with methotrexate or aminopterin.

How do the immunosuppressive or cancer chemotherapeutic agents cause malignant tumors? There are several possibilities. First, the drugs may be directly oncogenic. Second, the compounds may potentiate the effects of various environmental carcinogens such as tobacco, sunlight, or radiation. Third, the agents may cripple the surveillance function of the lymphoreticular system by which potentially malignant mutant cells are normally eliminated. Fourth, the weakened host defenses may permit oncogenic viruses to become established and cause malignant tumors.

While there is unequivocal evidence that the anticancer and immunosuppressive agents may cause de novo neoplasms their effects on patients with preexisting tumors are not so clearly defined. In organ transplant recipients with cancer there is a $41 \%$ likelihood of recurrence or metastases of the original tumor and a $6 \%$ incidence of unrelated de novo neoplasms. It is not possible to determine whether the former figure is merely a reflection of the natural history of the cancers or is contributed to by chronic immunosuppressive therapy.

In the case of advanced cancers treated with chemotherapy there are reports suggesting that, while the original cancer had been controlled, the long-term chemotherapy may have caused new cancers. No doubt there are numerous additional cases which have not been reported. The subject is a very complex one requiring consideration of the increased likelihood of a patient with one cancer developing a second neoplasm; the tendency for one form of cancer to change to another related type; and the influence of other therapeutic agents, such as radiotherapy, which may be oncogenic. In the present study the three most commonly used compounds were melphalan, cyclophosphamide, and busulfan-all alkylating agents. These have radiomimetic actions and are known to be mutagenic and carcinogenic in laboratory animals.

While cancer chemotherapy has had some notable successes, the overall results have been rather disappointing. These have been blamed on unresponsiveness of the tumor to a particular agent, or to the subsequent development of resistance by the cancer cells, or to the toxic effects of the compounds used. Another factor, which has received relatively scant attention, is the prolonged immunosuppressive effect of the agents when administered continuously. Could this be the explanation for the observation that a better objective response and longer survival was observed when chemotherapy was given intermittently rather than continuously?

The finding that cancer patients treated with chemotherapy may develop new tumors is more of academic than of practical importance and represents the price the patient has to pay for the hope of relief from the original cancer. Even so, several important lessons do emerge from this study. First, immunosuppressive agents should not be used in nonmalignant diseases, such as psoriasis or rheumatoid arthritis, unless all other forms of therapy have failed to provide relief. Second, in organ transplantation, donors with cancer should not be used except in cases with primary tumors of the central nervous system which seldom spread to other organs. 
Third, when a cancer arises in an immunosuppressed patient it may be useful to withdraw or reduce the immunosuppressive therapy in the hope that the host defenses may recover and resist the neoplasm.
Fourth, the studies emphasize the importance of the immune system in dealing with cance: and suggest that research on immunotherapy should be vigorously pursued.

\section{REFERENCES}

1. Penn, I., and Starzl, T. E.: Cancer. (In press). Transplant. Proc. 3:773, 1971.

2. Penn, I.: Malignant Tumors in Organ Trans- 4. Starzl, T. E., Penn, I., Putnam, C. W., Groth, plant Recipients. New York, Springer-Verlag, 1970. C. G., and Halgrimson, C. G.: Transplant. Rev. 3. Penn, I., Halgrimson, C. G., and Starzl, T. E.: $\quad 7: 112,1971$. 